\title{
N8 7-29457
}

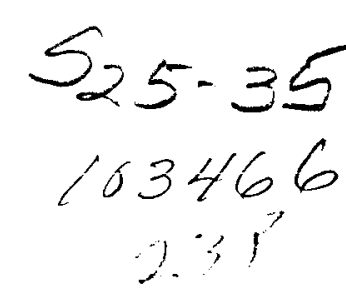

Particle fleld holography data reduction by Fourier transform analysis

Cecil P. Hess

James D. Trollnger

Spectron Development Laboratories, Inc.

3303 Harbor Blvd., Suite G-3, Costa Mesa, California 92626

\section{Abstract}

The size distribution of a particle field hologram is obtained with a Fourier transformation of the Fraunhofer diffraction pattern of the reconstructed hologram. Off-axis absorption holograms of particle fields with known characteristics were obtained and analyzed with a commercially available instrument. The mean particle size of the reconstructed hologram was measured with an error of $\pm 5 \%$, while the distribution broadening was estimated within $\pm 15 \%$. Small sections of a pulsed laser hologram of a synthetic fuel spray were analyzed with this method thus yielding a spatially resolved size distribution. The method yields fast and accurate automated analysis of particle field holograms.

\section{Introduction}

Pulsed laser holography has been a very powerful technique to characterize particle fields in complex environments. Examples are studies of pulverized coal combustion ${ }^{1}$, fuel sprays ${ }^{2}$, and explosions ${ }^{2}$. However, its use has been restricted because of the tedious effort required to 
reconstruct and analyze particle field holograms. The analysis of holograms has been performed by taking photographs at various planes of interest and then analyzing the photographs in computerized image analyzers. This suffers from various shortcomings. The most important are: photographic images have associated depths of field which lead to size ecrors; the process, even though more automated than the more primitive visual analysis, is still tedious. Recently, a technique using Fraunhofer diffraction analysis was introduced ${ }^{3}$. This technique bases the size measurement in the Fourier transform analysis of the light diffracted by an ensemble of particles illuminated by a laser beam. The direct analysis of a particle field with such method has been used quite extensively ${ }^{4}$. The logic proposed in reference [3] is that since the Fourier transform of the Fraunhofer diffraction pattern of an ensemble yields the size distribution, such transformation will also yield the size distribution if the particles are substituted by a particle field hologram.

This technique was tested ${ }^{3}$ in connection with on-line holograms, and its application was illustrated ${ }^{5}$ in the measurement of pulsating fuel sprays. There are, however, problems associated with reconstructing online holograms such as deterioration of the reference beam and confusion hetween the real and virtual inages.

The approach chosen by the authors and discussed in this paper was to use of $f$-axis holography in conjunction with Fourier transform analysis. Particle flelds of known characteristics were measured and compared. The results show that for Rossin Rammler size distributions the mean size was predicted very accurately $( \pm 5 \%)$, whlle larger errors were 
experienced in the broadening parameter $( \pm 15 \%)$. Some of these errors and inconsistencies were associated with instrument alignment and extraneous noise which, for the most part, can be eliminated, primarily by increasing the diffraction efficiency of the holograms.

The interface of pulsed laser holography with a Fourier transform analyzer of the diffracted light will greatly extend the capabilities of both. The former will benefit from fast almost-in-line data reduction. The later which is a much slower process when used in conjunction with holography will provide data unattainable until now. Examples are partfele shing in turbulent combustion phenomeña, where beam steering is a problem associated with light scattering techniques; explosions, where the events take place in microseconds; and time dependent sprays or other particle laden flows where it is of interest to resolve the time variation of the particle field distribution.

\section{Theoretical Discussion}

The amplitude scattered by a particle of radius a through an angle $\beta$ Is given by

$$
A=\frac{A_{i}{ }^{a J}{ }_{1}^{(k a \beta)}}{\beta}
$$

where $A_{i}$ is the illuminating amplitude

$J_{1}$ is the Bessel function of the first kind, and $k=2 \pi / \lambda$ and $i t$ is assumed that

a $>\lambda \lambda$ the wavelength of $1 \mathrm{ight}$. 
If a distribution of particle sizes $f(a)$ exists, then the contribution by those of size between $a$ and $a+d a$ is to the amplitude at an angle $B$ is

$$
\frac{f(a) d a \quad a J_{1}(k a \beta)}{\beta}
$$

and the total amplitude in that direction is

$$
A_{t}=\frac{A_{i}}{\beta} \int_{0}^{\infty} f(a) a J_{1}(k a \beta) d a
$$

What is sought in particle sizing measurement is $f(a)$ which is given by ${ }^{6}$

$$
f(a)=\frac{-2}{k a} \int_{0}^{\infty} \beta J_{1}(k a \beta) Q(\beta) Y_{1}(k a \beta) d \beta
$$

where $Y_{1}$ is the Neumann function and

$$
Q(B)=\frac{d}{d B}\left[\pi k^{3} B^{3} \frac{I}{I_{0}}\right]
$$


The above expression has been approximated numerically and is the basis for particle sizing instruments based on a measurement of the angular spectrum of scattered light. There is a one-to-one correspondence between the angular spectrum and the Fourier transform of the scattered light, and the latter is what is usually measured.

If the wavefront scattered by the collection of particles is stored in a hologram as an intermediate step and reconstructed later for analysis, it becomes of concern how the ultimate wavefront may differ from the original one.

The hologram can be represented by a transfer function. The amplitude of light reconstructed from the hologram can be written ${ }^{7}$ (using only one of the two dimensions)

$$
A_{t}(\beta / \lambda)=A_{i}(\beta / \lambda) * T(B / \lambda)
$$

where

$$
T(B / \lambda)=\int_{-\infty}^{\infty} t(y) \exp \left[j 2 \pi \frac{\beta}{\lambda} y\right] d y
$$

The convolution has characteristics which can both narrow and broaden the spectrum. If the hologram is too small, then the spectrum will be broadened by its own diffraction. If it is large and perfect, then $T$ is effectively unity. In practice, the transfer function, $T$, is more likely to decrease gradually with $\beta$ as the film MTF decreases with 
frequency. The effect is to bias the distribution of $f(a)$ to larger particles. These above relations show that with good practice the data derived with holography as an intermediate step can accurately emulate that using the Fourier Transform directly, and they could be used to correct for holograms not having a unity transfer function.

\section{Experimental approach}

The approach chosen in this study was that of comparing the size distribution obtained by analyzing a known particle field with that of a hologram of the same particle field. To simplify the experiment, the particle field chosen was stationary thus allowing the use of a cw He-Ne laser for the acquisition of the hologram. The Fourier transformation was performed by a Malvern 2600 particle analyzer. A description of the apparatus and particle fields is now given.

\section{Holographic system}

The holographic system was a breadboarded, off-axis, through field system, having separate object and reference beams, as seen in Figure 1. The object beam was disturbed by a particle field, while the reference beam was undisturbed. The two beams interfere when they overlap on a holographic film plate.

A $25 \mathrm{~mW}$ Helium Neon Laser (Spectra Physics Model 107) was used as the illumination source. The beam was split with an uncoated glass beamsplitter (Newport Corporation \#20B10NC.1). A split ratio of 4:1 (reference to object beam) was chosen to provide good reconstruction 
efficiency while remaining in the linear region of the film. The ratio was adjusted by varying the angle of incidence to the beamsplitter, thus changing the reflectivity.

The reference beam was expanded using a $5 x$ microscope objective and spatially filtered with a 40 micron pinhole. It was collimated by a 380 $\mathrm{mm}$ focal length achromatic lens and apertured to a $10 \mathrm{~mm}$ diameter nearly uniform intensity beam. The collimated reference beam was then directed to the holographic plate, where its peak intensity was measured at 28 $\mu \mathrm{w} / \mathrm{mm}^{2}$.

A $10 \mathrm{x}$ microscope objective and a 25 micron pinhole were used to expand and filter the object beam. An achromatic collimating lens, with a $125 \mathrm{~mm}$ focal length, produced a Gaussian object beam with a diameter of $10 \mathrm{~mm}\left(1 / \mathrm{e}^{2}\right)$. The object beam illuminated a particle field which was 1maged on the holographic plate by 1:1 magnification lens system. This 1maging system consisted of two $50 \mathrm{~mm}$ 0lympus Lenses positioned back-toback, connected by bellows. It collected the forward scattered radiation and the unscattered radiation and relayed them to the holographic plate. The object beam peak intensity was measured to be $6.4 \mu \mathrm{w} / \mathrm{mm}^{2}$.

\section{The Fourier Transform Analyzer}

The receiver of a Malvern 2600 was used to collect and analyze the Fraunhofer diffraction field. A Fourier transform lens, in the receiver, collects both the forward scattered and unscattered radiation and focuses it onto a concentric photodiode array. The scattered radiation is transformed to a series of diffraction rings at the focal plane of the lens. Thirty concentric half-ring diodes receive these data 
which are transferred to a minicomputer for processing. The unscattered radiation is focused on a pinhole at the focal plane of the receiving lens. This radiation illuminates a separate diode, indicating the amount of unscattered radiation.

Figure 2 shows a photograph of the holographic system interfaced to the Malvern recelver which is interfaced to a microprocessor based data reduction system. The holographic recording/reconstruction system was designed to produce holograms capable of simulating the Malvern transmitter beam illuminating a particle field.

\section{Particle fields}

Two particle flelds were used throughout these experiments. The first was a calibration photomask (Laser Electro-0ptics Ltd. ${ }^{8}$ \#RR 503.0-0.08-102.CF) which contains a known size distribution of circular particles. The second was a particle field hologram of a spray of synthetic fuel (SRCII) acquired in a previous program using a pulsed laser holographic system.

The photomask was chosen for two primary reasons:

1. It provided a known and stationary particle field of which holograms could be taken with a cw laser.

2. Both the photomask and holograms of the photomask could be analyzed and compared against an absolute invariable distribution. 
The calibration standard photomask consisted of a 2-dimensional sample array of 10,000 circular discs of chrome deposited on a glass substrate. The discs are randomly oriented in an $8 \mathrm{~mm}$ diameter sample area. The photomask contalns a Rossin Rammler size distribution with $\overline{\mathrm{X}}=50 \mu \mathrm{m}$ and a width parameter of 3.0 (Figure 3 ).

The particle field hologram described here containing a synthetic fuel spray was taken previously using a pulse ruby laser holocamera. The receiving optics provided a premagnification of $3: 1$. It has previously been determined, through existing reconstruction techniques, that the mean size of the spray droplets in this hologram lies in the range from 50 to $100 \mu \mathrm{m}$ (depending on location) which will be interpreted as 150 to $300 \mu \mathrm{m}$ on the magnified 1mage of $3: 1$.

Procedure to align the Malvern receiver

The reconstructed image of the hologram must be collected by the recelving lens (we used the $300 \mathrm{~mm}$ in all the tests) and focused on the diode array. Since the receiving lens of the Malvern has no focus adjustment, it is important that the reconstructed unscattered radiation be collimated and, therefore, produce a sharp focus on the hole in front of the center diode.

The receiver was then steered until its optical axis was parallel to the reconstructed unscattered 1ighty. It was then translated vertically and horizontally until the light beam entered through the center of the lens.

The final fine adjustments were made with the adjustment screws of the receiver. 
Signal level requirement

We established that the radiation power of the reconstructed holographic image was typically between .15 mw and .25 uw. This can be obviously increased using a more powerful laser or increasing the diffraction efficiency of the hologram (using phase holograms). Since the Malvern apparatus uses a 2.5 mw laser, we had to establish the performance of the photodiode array at low power. The procedure consisted in 1lluminating the photomask with different levels of laser power. For this purpose, a variable neutral density filter was placed in front of the laser such that the total power of the light incident on the receiver varied between $50 \mu \mathrm{w}$ and $2.5 \mathrm{mw}$. Under each one of these laser power levels we obtained a size distribution using the Rossin Rammler and the model independent softwares. Power levels of $100 \mu \mathrm{w}$ and more produced the same size distribution of the photomask. Power levels below $50 \mu \mathrm{w}$ were not acceptable. It was also necessary, in order to work with the low power levels, to darken the room to reduce background radiation. Based on these results, it was expected that the typical 150 w signal obtained from the reconstructed image would be adequate. Let us point out again that higher signal levels could be attained by reconstructing with a more powerful laser or by producing more efficient holograms. Obviously, increasing the diffraction efficiency without compromising the linearity would be the preferred choice since part of the noise comes from scatterers on the emulsion. 


\section{Results}

Three particle fields were Fourier transform analyzed: (1) a calibration reticle or photomask, (2) a reconstructed image of a hologram of such photomask, (3) reconstructed image of a spray hologram.

The two different softwares provided by Malvern were used where appropriate. The first finds the best fit to a Rossin Rammler distribution, while the second is supposed to be independent of a preconceived model. The values of $X$ and $N$ (defined below) were obtained with the Rossin Rammier distribution and are indicated in the figure legends of the model independent data.

The photomask data are summarized on Table 1 . This table shows the data obtained by analyzing the scattered light of the reconstructed holographic image of the photomask, and also the light scattered from the direct photomask. The parameters describing the size distribution are $\bar{X}$ and $N$ which are the two parameters of the Rossin Rammler distribution which is given by:

$$
V=e^{-(x / \bar{X})^{N}}
$$

where $V$ is the volume fraction of drop material occurring in drops of dfameter greater than $X$. The volume distribution is given by:

$$
\frac{d v}{d x}=\frac{N x^{N-1}}{\bar{X}^{N}} e^{-(X / \bar{X})^{N}}
$$




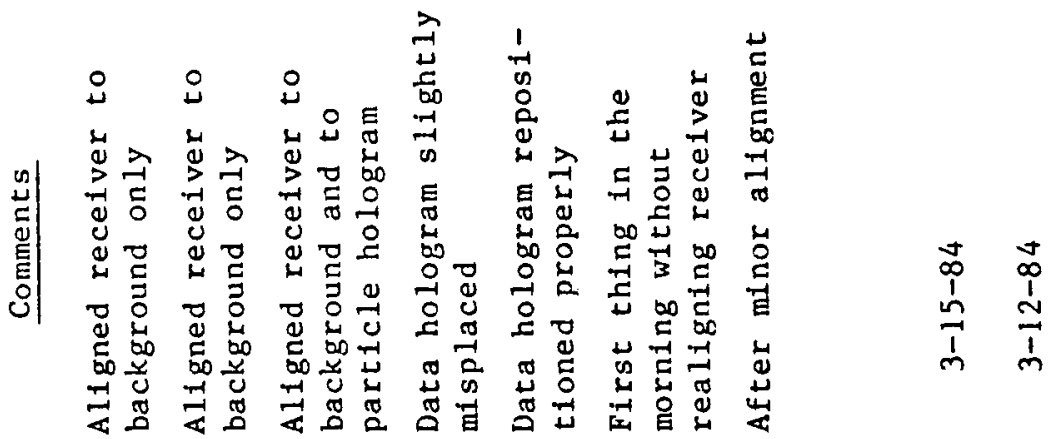

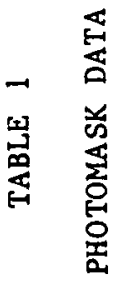

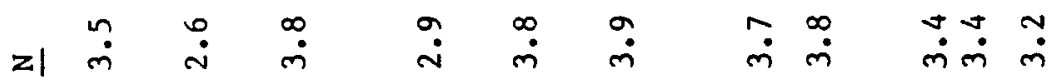

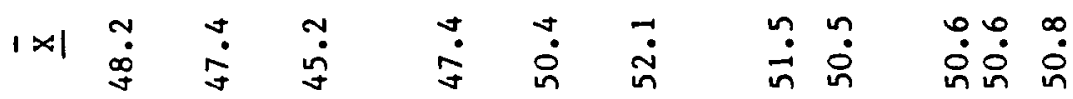



이 సें సें लें लें लें ले ले

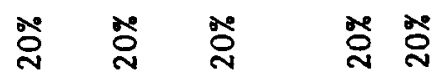

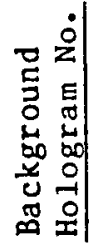

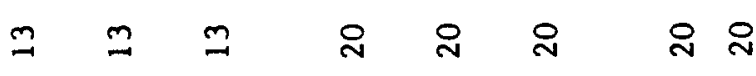

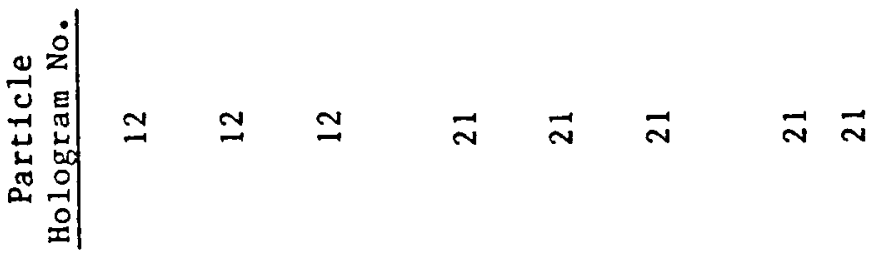

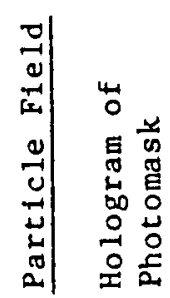


The SMD is related to $\bar{X}$ and $N$ by the expression:

$$
\operatorname{SMD}=\frac{\bar{X}}{\Gamma\left(1-\frac{1}{N}\right)}
$$

To 1llustrate the effect of $\bar{X}$ and $N$ on the size distribution, Figure 4 shows two volume distributions corresponding to $\bar{X}=50$ and $N=3$ and 3.5. Large values of $\mathrm{N}$ correspond to narrow distributions.

Light Energy Distribution

The light energy distribution of the photomask and 1ts holographic reconstructed 1mage were also analyzed (Figure 5). One of the difficulties encountered in processing the holographic data stemmed from the inaccuracy in repositioning the hologram. Some of the unscattered radiation would miss the central diode and illuminate the second diode. This will show as a bin in the large size band. Figure 5 illustrates this problem. Notice the large energy level measured on Bin \#15 of the holographic data. In general, however, the agreement shown between the light energy distribution produced by scattering from the real photomask and the reconstructed hologram is quite good. This probably justifies the results obtained with the Rossin Rammler software. Namely, $\bar{X}=51.5, N=3.7$ for the reconstructed hologram, and $\bar{X}=50.6$, $N=3.4$ for the direct photomask. It is not, however, clear why the $N$ value of the hologram is larger (3.7) than the corresponding photomask 
(3.4). A large value of $\mathrm{N}$ is associated with a narrow distribution and it is not clear that this should be the case, given that the hologram has additional noise which should broaden the distribution. This might be the result of an artifact of the inversion scheme software.

\section{Spray Hologram Data}

A hologram of a spray field of synthetic fuel (SRCII) previously acquired with a pulsed ruby laser was then analyzed. This hologram was obtained using (almost) uniform object and reference beams and a premagnification of 3 . To best simulate the conditions normally used by the Malvern, a Gaussian beam of $6 \mathrm{~mm}$ diameter $\left(1 / \mathrm{e}^{2}\right)$ was used for reconstruction. The reconstructed image had a total power of about $0.25 \mathrm{mw}$ and a (almost) Gaussian intensity profile. The divergence of the 1lluminating reconstruction wave was controlled to produce a well collimated reconstructed wave.

Three regions of the hologram were reconstructed and analyzed with the receiver, and a region without spray was used to obtain the background information. These data are shown on Figure 6 . It is important to realize that since the hologram was acquired with a $3: 1$ magnification, the actual droplet size is $1 / 3$ of that shown.

Figure 6 shows photograph of a given plane of the 3-D hologram, and inserts corresponding to the locations where data were acquired. Notice that the mean value changed from $285 \mu \mathrm{m}$ (actual size $95 \mu \mathrm{m}$ ) at the edge of the spray to $165 \mu \mathrm{m}$ (actual size $55 \mu \mathrm{m}$ ) at the center. This is the typical trend of sprays formed by simple pressure nozzles in which more large droplets are found at the edge of the spray. 


\section{Acknowledgments}

This work was supported by the Air Force Wright Aeronautical Laboratories, Aero Propulsion Laboratory, Air Force Systems Command, U. S. Air Force, Wright-Patterson AFB, Ohio 45433, under contract number F337615-83-C-2372 managed by Lt. Abdollah S. Nejad.

The authors are also grateful to the Environmental and Energy Research Corporation for lending the Malvern particle analyzer and providing technical support. They also appreciate the fine photographic work provided by Robert Nichols.

\section{References}

1. Trolinger, J. D. and Heap, M. P., Applied Optics, 18, 1757 (1979).

2. Wuerer, J. E., Oeding, R. G., Poon, C. C., and Hess, C. F., AIAA 20th Aerospace Sciences Meeting, AIAA-82-0236 (1982).

3. Ewan, B.C.R., Applied Optics, 19, 1368 (1979).

4. Swithenbank, J., Beer, J. M., Taylor, D. S., Abbot, D., and McCreath, G. C., AIAA 14th Aerospace Sciences Meeting, AIAA-76-69 (1976).

5. Ewan, B.C.R., Swithenbank, J., and Sorusbay, D., Optical Engineering, $23,620(1984)$. 
6. Abbiss, J. B., "Theoretical Aspects of the Determination of ParticleSize Distributions from Measurements of Scattered Light Intensity." Royal Aircraft Establishment TR70151, August 1970.

7. Goodman, J. W., Introduction to Fourier Optics, p.52, McGraw Hill (1968).

8. Hirleman, E. D., ASME Gas Turbine Meeting, ASME Paper No. 83-GT-232. 


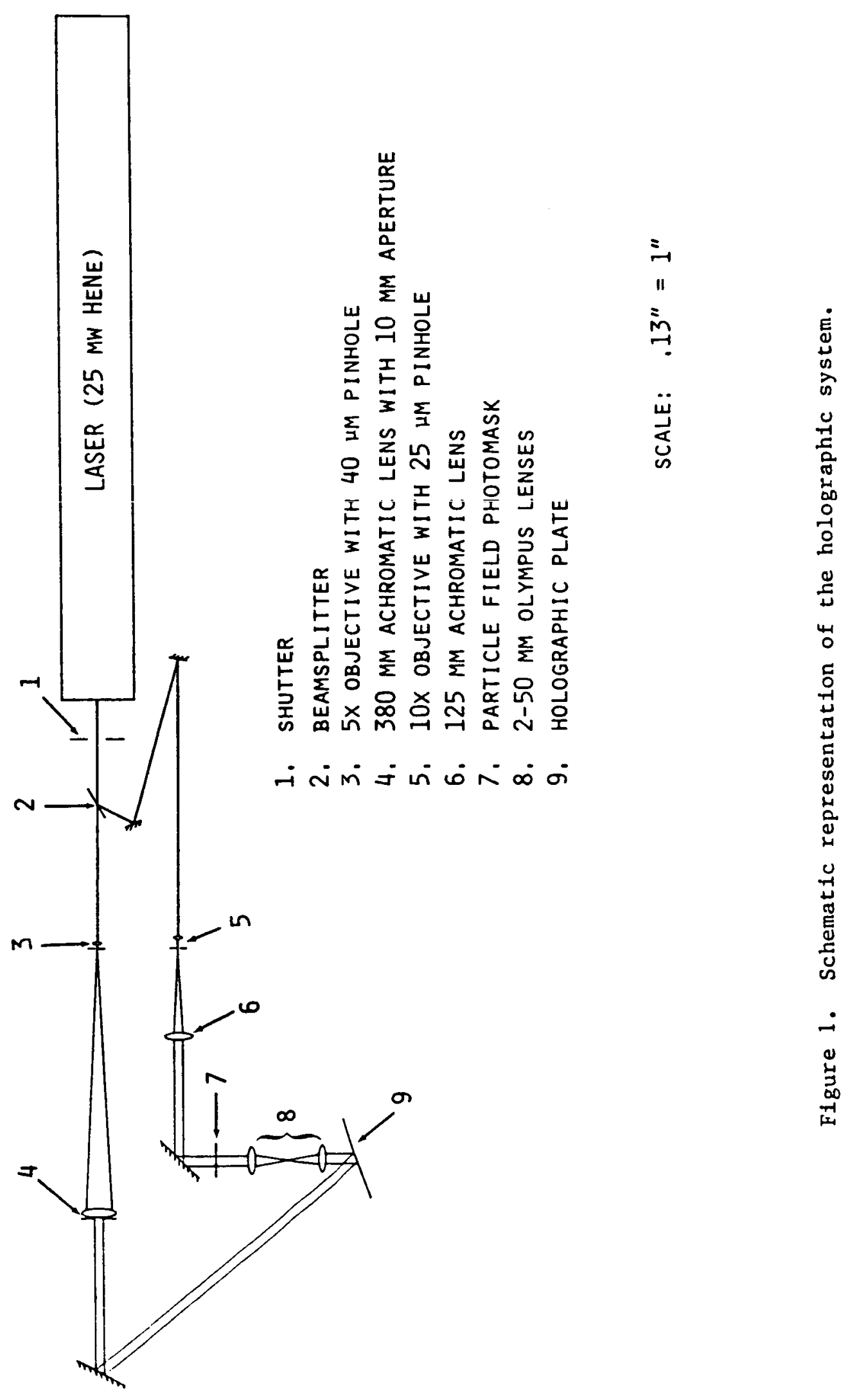




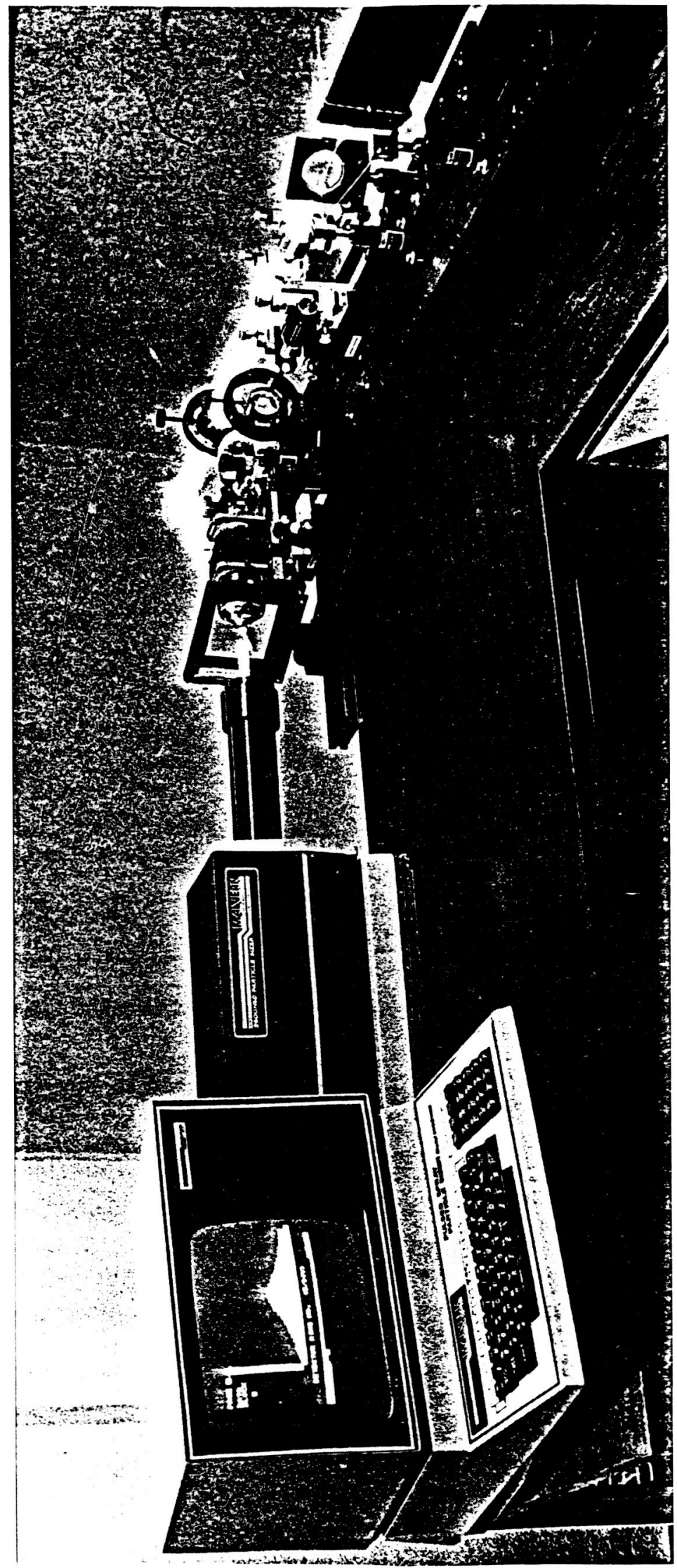

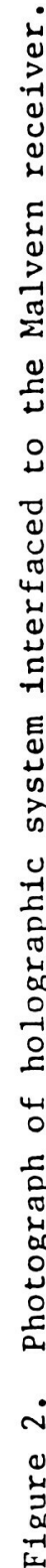



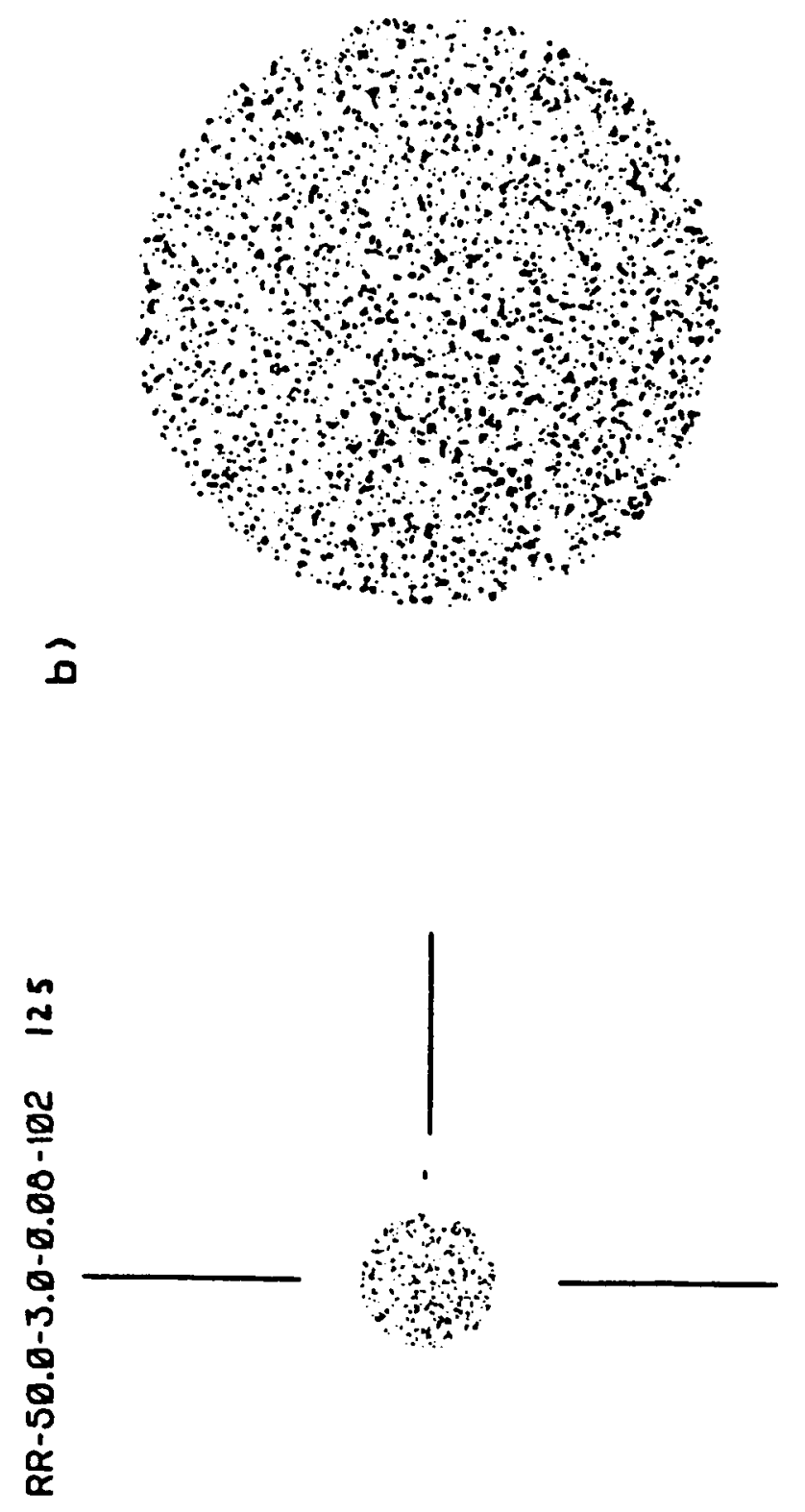

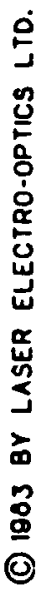

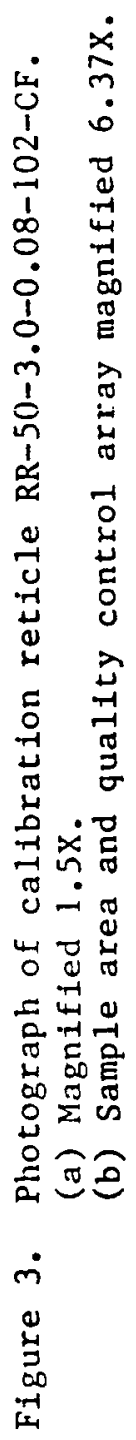




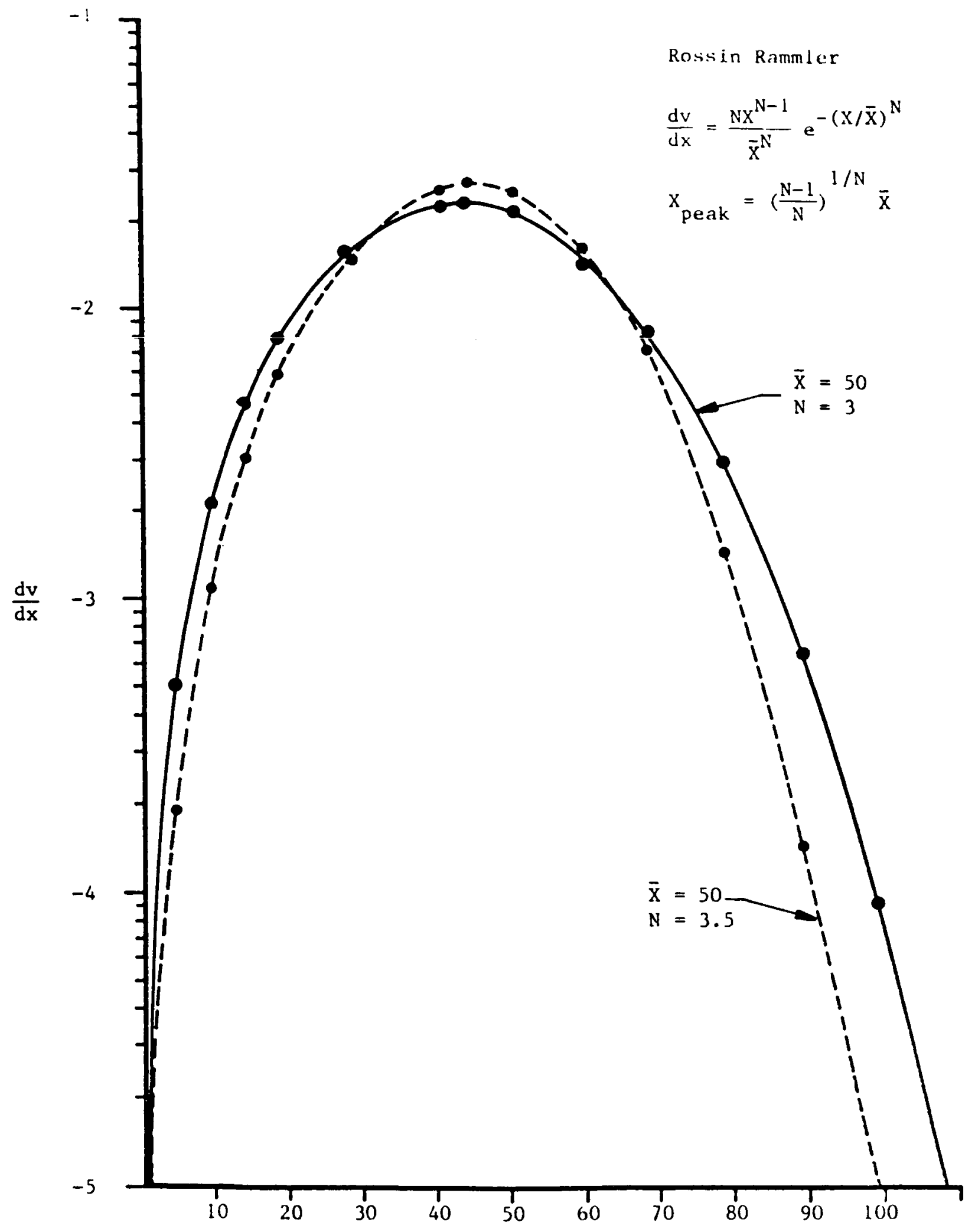

Figure 4. Effect of $\mathrm{N}$ on Rossin Rammler volume distribution. 


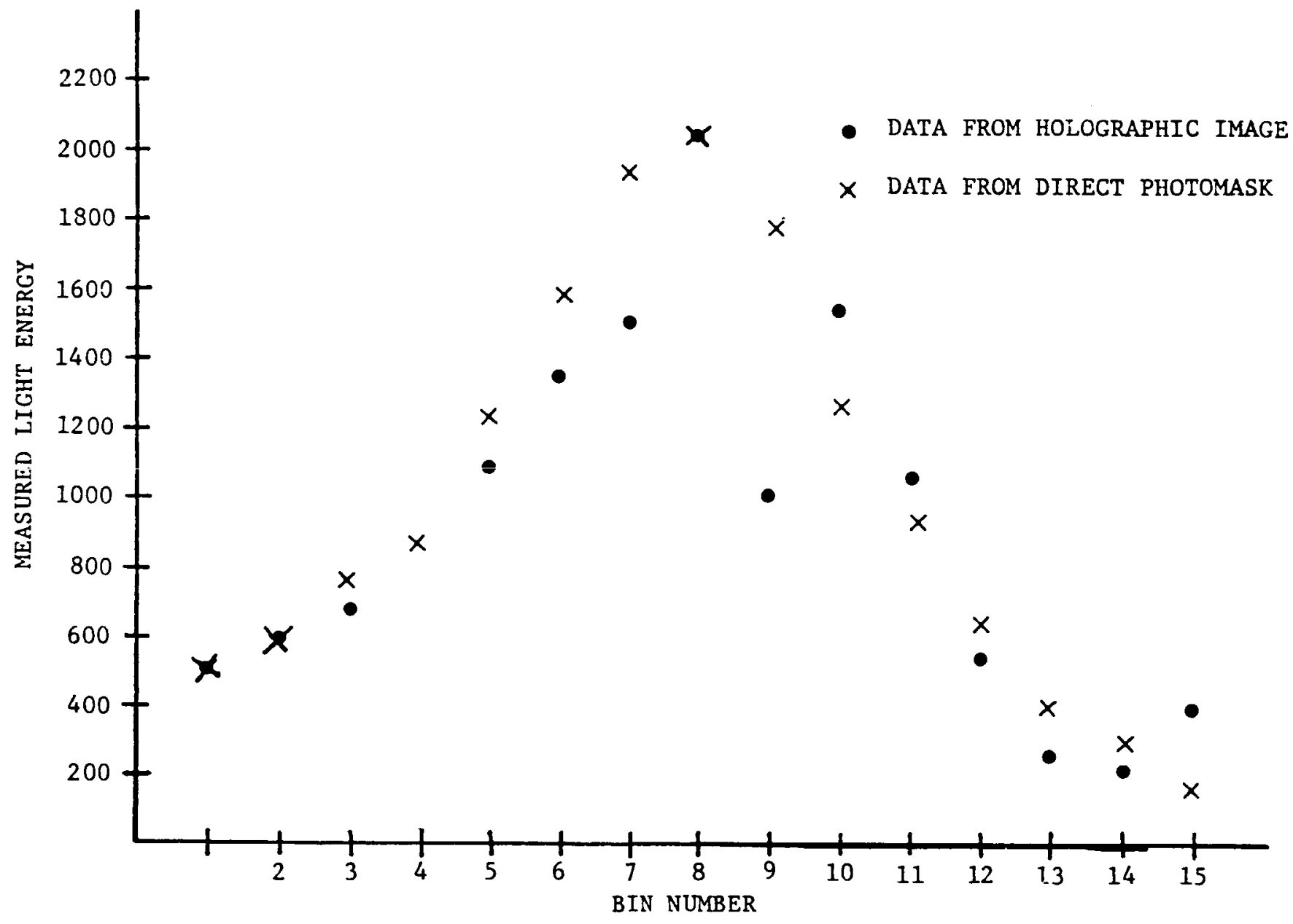

Figure 5. Measured energy levels of the lights scattered by photomask and hologram of photomask on 15 diodes of Malvern receiver. The corresponding Rossin Rammler parameters are $\bar{X}=50.6$, $\mathbf{N}=3.4$ (direct photomask), and $\bar{x}=51.5, N=3.7$ (reconstructed hologram of photomask). 


\section{FOURIER DIFFRACTION ANALYSIS}

\section{OF A FUEL SPRAY HOLOGRAM}

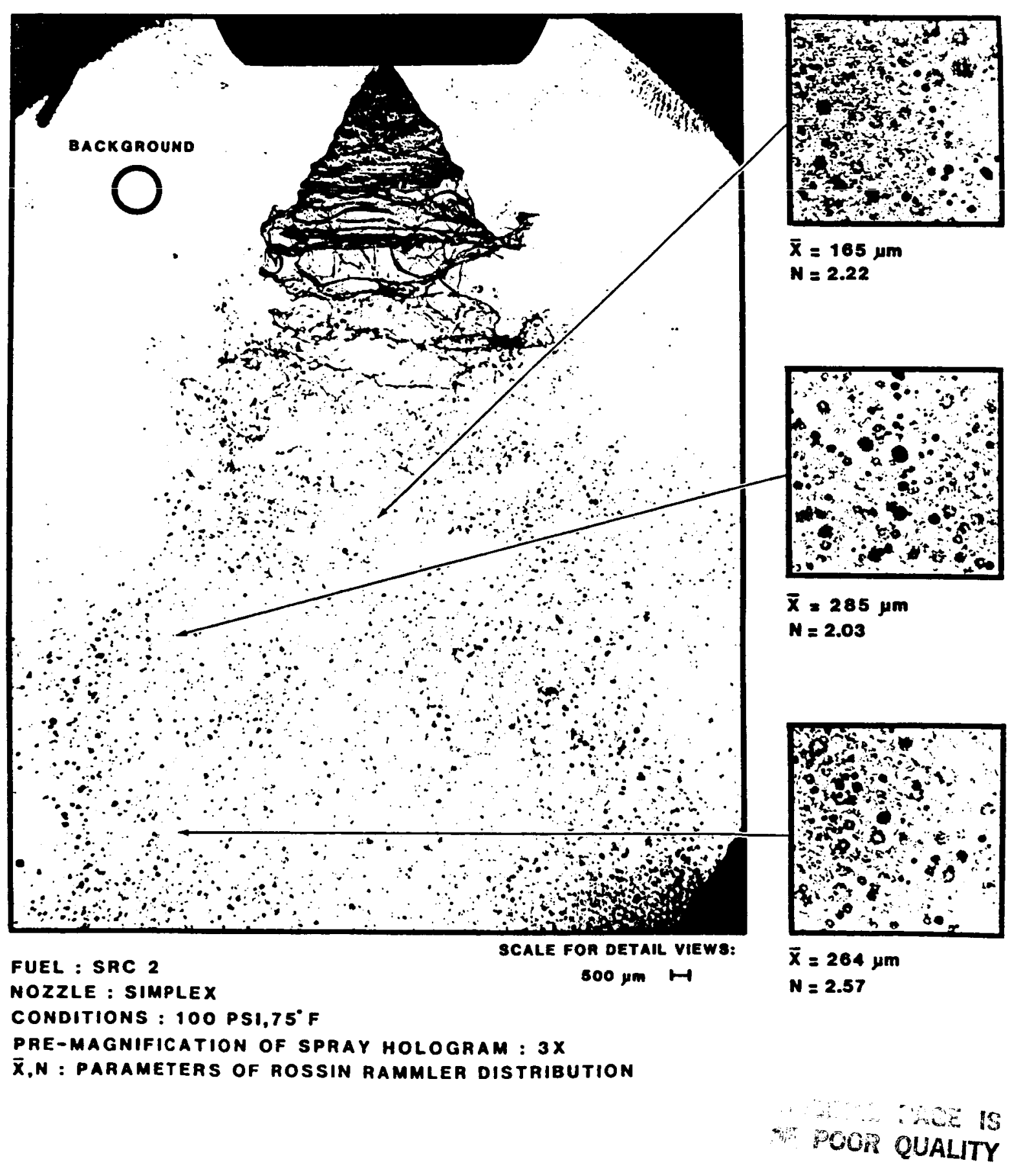

Figure 6. Fourier transform reduction of a spray hologram obtained with a pulsed ruby laser. 
KEY WORDS

1. Particle field holography

2. Automated data reduction 
1 Schematic representation of the Holographic System

2 Photograph of holographic system interfaced to the Malvern receiver.

3 Photograph of calibration reticle RR-50-3.0-0.08-102-CF. (a) Magnified 1.5X. (b) Sample area and quality control array magnified $6.37 x$.

4 Effect of $N$ on Rossin Rammler volume distribution.

5 Measured energy levels of the lights scattered by photomask and hologram of photomask on 15 diodes of Malvern receiver. The corresponding Rossin Rammler parameters are $\mathbb{X}=50.6, N=3.4$ (direct photomask), and $X=51.5, N=3.7$ (reconstructed hologram of photomask).

6 Fourier transform reduction of a spray hologram obtained with a pulsed ruby laser. 